\title{
Sylvie Ballestra-Puech, Templa serena. Lucrèce au miroir de Francis Ponge
}

Sara Arena

\section{(2) OpenEdition}

1 Journals

\section{Edizione digitale}

URL: http://journals.openedition.org/studifrancesi/616

DOI: 10.4000/studifrancesi.616

ISSN: 2421-5856

\section{Editore}

Rosenberg \& Sellier

\section{Edizione cartacea}

Data di pubblicazione: 1 aprile 2015

Paginazione: 194

ISSN: 0039-2944

\section{Notizia bibliografica digitale}

Sara Arena, «Sylvie Ballestra-Puech, Templa serena. Lucrèce au miroir de Francis Ponge », Studi Francesi [Online], 175 (LIX | I) | 2015, online dal 01 avril 2015, consultato il 18 septembre 2020. URL : http:// journals.openedition.org/studifrancesi/616; DOI : https://doi.org/10.4000/studifrancesi.616

Questo documento è stato generato automaticamente il 18 settembre 2020.

\section{(c) $(1) \&$}

Studi Francesi è distribuita con Licenza Creative Commons Attribuzione - Non commerciale - Non opere derivate 4.0 Internazionale. 


\title{
Sylvie Ballestra-Puech, Templa serena. Lucrèce au miroir de Francis Ponge
}

\author{
Sara Arena
}

\section{NOTIZIA}

SYLVIE BALLESTRA-PUECH, Templa serena. Lucrèce au miroir de Francis Ponge, Genève, Droz, 2013, pp. 470.

1 In questo saggio denso e complesso, Sylvie Ballestra-Puech indaga l'influenza esercitata su Ponge dal celeberrimo poema di Lucrezio, a cui il poeta novecentesco, autore di un suo personalissimo De natura rerum, era accomunato dall'importanza attribuita all'osservazione razionale della natura.

2 A dimostrare la contiguità tra le due opere non sono solo i numerosi rimandi espliciti, le citazioni dirette e indirette, le suggestioni, le riprese di immagini e gli echi più sottili, peraltro finemente analizzati nel volume, ma anche analogie di fondo nel pensiero dei due autori.

3 Tra queste, la prima su cui Sylvie Ballestra-Puech concentra la sua attenzione è l'interesse rivolto al fenomeno linguistico, all'origine del linguaggio verbale e al suo rapporto con gli oggetti: se per Lucrezio il suono, nato dall'emozione, conserva la capacità di suscitarla, anche per Ponge nominare qualcosa significa, sia pur in modo tortuoso, esprimere e glorificare la materia. Tra mondo e linguaggio vi è inoltre un'analogia di funzionamento, in quanto entrambi si costruiscono attraverso combinazioni di elementi semplici quali gli atomi e le lettere. A questa visione della lingua vanno ricondotte anche alcune tecniche di scrittura, come la paronomasia, le figure di suono, lo sviluppo di mots-thème, che fanno dell'opera di Ponge «moins [le] récit d'une genèse du monde [que la] création d'un monde verbal homologue au monde des choses» (p. 67). 
4 A fornire a Ponge un'ulteriore chiave di accesso al poema di Lucrezio fu, ricorda l'A., il filosofo Bernard Groethuysen, che gli fece conoscere il titolo dell'opera di Cardano De varietate rerum. Nell'osservazione del mondo, il poeta era infatti soprattutto colpito dalla capacità della materia di dare luogo a oggetti tanto diversi a partire da alcuni limitati elementi, varietà a cui corrisponde nell'opera una vera e propria poetica della variazione che avvicina molte pagine di Ponge alla tipologia musicale della fuga (E. Noulet, cit. a p. 131) e che giustifica l'ammirazione manifestata dall'autore anche per le Metamorfosi di Ovidio.

5 Nel suo continuo dialogo con l'opera lucreziana Ponge si inserisce inoltre, a suo modo, anche nel dibattito sviluppatosi nei secoli attorno all'opera e alla figura di Lucrezio. Ponge prende in particolare posizione contro l'interpretazione di Benjamin Logre che nel 1946, aggiungendosi a una lunga schiera di detrattori, pubblicava L'Anxiété de Lucrèce, interpretazione clinica dell'opera di Lucrezio in cui, forzando non poco il testo lucreziano, tentava di avvalorare la tesi di San Gerolamo di un Lucrezio affetto da disturbi psichici che lo avrebbero condotto fino al suicidio.

6 Sylvie Ballestra-Puech approfondisce infine la poetica della contemplazione, a cui la citazione lucreziana del titolo, templa serena, allude. Ogni oggetto, anche minimo, è un mondo a sé e al tempo stesso contiene il mondo, poiché costituisce un frammento della sua tessitura. Come i quadri di Braque, non a caso amato dall'autore del Parti pris des choses, i testi di Lucrezio e quelli di Ponge sono altrettanti templa, ossia porzioni di mondo, frammenti in cui «l'océan de la matière devie[nt] objet de contemplation au lieu d'être ressenti comme une menace d'annihilation» (p. 208). 